\title{
Asymmetrical bonding in liquid Bi disentangled by inelas- tic X-ray scattering
}

\author{
Masanori Inui ${ }^{1, *}$, Yukio Kajihara ${ }^{1}$, Shuji Munejiri ${ }^{1}$, Shinya Hosokawa $^{2}$, Ayano Chiba $^{3}$, Koji \\ Ohara $^{4}$, Satoshi Tsutsui ${ }^{4}$, and Alfred Q. R. Baron ${ }^{5,4}$ \\ ${ }^{1}$ Graduate School of integrated Arts and Sciences, Hiroshima University, Higashi-Hiroshima, Hi- \\ roshima 739-8521, Japan \\ ${ }^{2}$ Department of Physics, Graduate School of Science and Technology, Kumamoto University, Ku- \\ mamoto 860-8555, Japan \\ ${ }^{3}$ Department of Physics, Keio University, Yokohama 223-8522, Japan \\ ${ }^{4}$ Japan Synchrotron Radiation Research Institute, SPring-8, Sayo-gun, Hyogo 679-5198, Japan \\ ${ }^{5}$ Materials Dynamics Laboratory, RIKEN SPring-8 Center, Sayo-gun, Hyogo 679-5148, Japan
}

\begin{abstract}
The structure of liquid $\mathrm{Bi}$ has been debated in relationship with the Peierls distortion, as crystalline Bi takes A7 structure. A recent $a b$ initio molecular dynamics simulation for liquid Bi predicted a flat-topped profile of the acoustic dispersion curve. To confirm the prediction, we have carried out inelastic x-ray scattering (IXS) for liquid Bi. The dynamic structure factor obtained by the IXS exhibits a distinct inelastic excitation of the longitudinal acoustic mode up to $14 \mathrm{~nm}^{-1}$ and the dispersion curve of the excitation energy obtained by the memory function analysis becomes a flat-topped one. We found that a linear chain model including the interatomic interaction with the second nearest neighbors can explain the flat-topped profile. The result suggests that the anomalous dispersion curve in liquid $\mathrm{Bi}$ arises from local anisotropy related to the Peierls distortion in the crystalline phase. .
\end{abstract}

\section{Introduction}

Bi belongs to the same group as As in the periodic table of elements, and the local structure of liquid Bi has been discussed from a view-point of the Peierls distortion [1]. The Peierls distortion is realized in a A7 crystalline structure type comprising three short and three long bonds in a distorted simple cubic lattice to gain the electronic energy.

The structure factor $S(Q)$ of liquid $\mathrm{Bi}$, where $Q$ is momentum transfer, exhibits a shoulder at high momentum transfer side of the first maximum (see Fig. 3) [2-4], in contrast to a symmetrical profile of simple liquid metals. The origin of the shoulder in $S(Q)$ of liquid Bi was theoretically studied and it was found that an effective pair potential with a ridge in the repulsive component could reproduce the shoulder [5,6]. Later, an ab initio molecular dynamics (AIMD) simulation for liquid Bi [7] revealed that longer and shorter bonds produce a distinct shoulder at the first peak in $S(Q)$.

Atomic dynamics in liquid Bi had been studied by inelastic neutron scattering (INS) experiments. Most recently, Sani et al. [8] carried out INS experiments at $Q \leq 6 \mathrm{~nm}^{-1}$ and

\footnotetext{
*e-mail: masinui@hiroshima-u.ac.jp
} 
reported the dynamic structure factor $S(Q, E)$, where $E$ is energy transfer, with good statistics. The $S(Q, E)$ exhibits the inelastic excitations of the acoustic mode dispersing with $Q$. However, this result did not fix conflicting INS results obtained in 1980's: Dahlborg and Olsson $[9,10]$ reported no distinct inelastic excitation of the acoustic mode in $S(Q, E)$ at $Q>6$ $\mathrm{nm}^{-1}$, while Shibata et al. [11] reported experimental observation of the acoustic excitations up to $12 \mathrm{~nm}^{-1}$. Later Dzugutov and Dahlborg [5] reported that there appears no distinct acoustic excitation at $Q>6 \mathrm{~nm}^{-1}$ with a classical molecular dynamics simulation (CMD), consistent with the INS results by Dahlborg and Olsson.

Contrary to the CMD result, Souto et al. [7] reported that inelastic excitations of the acoustic mode survive up to $14 \mathrm{~nm}^{-1}$ in $S(Q, E)$ obtained by an ab initio molecular dynamics (AIMD) simulation. Furthermore the AIMD simulation for liquid Bi predicted an intriguing dispersion curve of the acoustic mode with a flat-topped profile, which is much different from a sinusoidal profile in simple liquid metals [12]. To investigate the behavior of the acoustic excitation in liquid $\mathrm{Bi}$, we carried out inelastic x-ray scattering (IXS) experiments. A high-resolution IXS technique allows us to avoid the kinematic constraints of INS and to obtain good spectra out to large energy transfer. The technique is also advantageous for measurements at extreme conditions such as high temperatures and high pressures owing to a small beam size of approximately $0.1 \mathrm{~mm}$ in diameter. Details of the IXS results have already been reported in Ref.[13]. In this article, we report the results of the acoustic mode in liquid Bi briefly and present results obtained by $S(Q, E)$ near the first peak position of $S(Q)$ that are not published.

\section{Experimental procedure}

The IXS experiments were conducted at the high-resolution IXS beamline (BL35XU) at SPring-8 in Japan [14]. Backscattering at the Si (11 11 11) reflection provided a beam of approximately $10^{10}$ photons/sec in a $0.8 \mathrm{meV}$ bandwidth onto the sample. The energy of the incident beam and the Bragg angle of the backscattering were $21.747 \mathrm{keV}$ and approximately $89.98^{\circ}$, respectively. The spectrometer resolution was approximately $1.5 \mathrm{meV}$ depending on the analyzer crystal, and it was experimentally determined by scattering from polymethyl methacrylate (PMMA). $Q$ resolution, $\Delta Q$, was set to be 0.45 and $1.0 \mathrm{~nm}^{-1}$ (full width) for $Q \leq 11$ and $Q>11 \mathrm{~nm}^{-1}$, respectively.

$\mathrm{X}$-ray diffraction measurements using monochromatized high-energy $\mathrm{x}$-ray were carried out at BL04B2/SPring-8 [15], to obtain $S(Q)$. Details of the data processing are described in Ref. [13].

Liquid Bi of $99.999 \%$ purity was mounted in a single-crystalline sapphire cell of Tamuratype [16] that was carefully machined to provide a $0.04 \mathrm{~mm}$ sample thickness. The cell was placed in "Marburg" chamber [17]. IXS spectra were measured at 573 and $1023 \mathrm{~K}$ [18] in pure $\mathrm{He}$ atmosphere at $0.1 \mathrm{MPa}$. The background spectra were measured at $573 \mathrm{~K}$ with an empty cell. After the absorption correction, the backgrounds were subtracted from the data, and $S(Q, E) / S(Q)$ were obtained. More details were described in Ref. [13].

\section{Computer simulations}

AIMD simulations were performed at $573 \mathrm{~K}$ using Quantum ESPRESSO package [19], which is based on the density functional theory, plane waves and pseudopotentials. The generalized gradient approximation(GGA) [20] was adopted for the exchange-correlation energy. We used the norm-conserving pseudopotential Bi.pbe-hgh.UPF [21] for the electron-ion interaction with five valence electrons, $6 \mathrm{~s}^{2} 6 \mathrm{p}^{3}$. The plane-wave cutoff energy for the electronic 


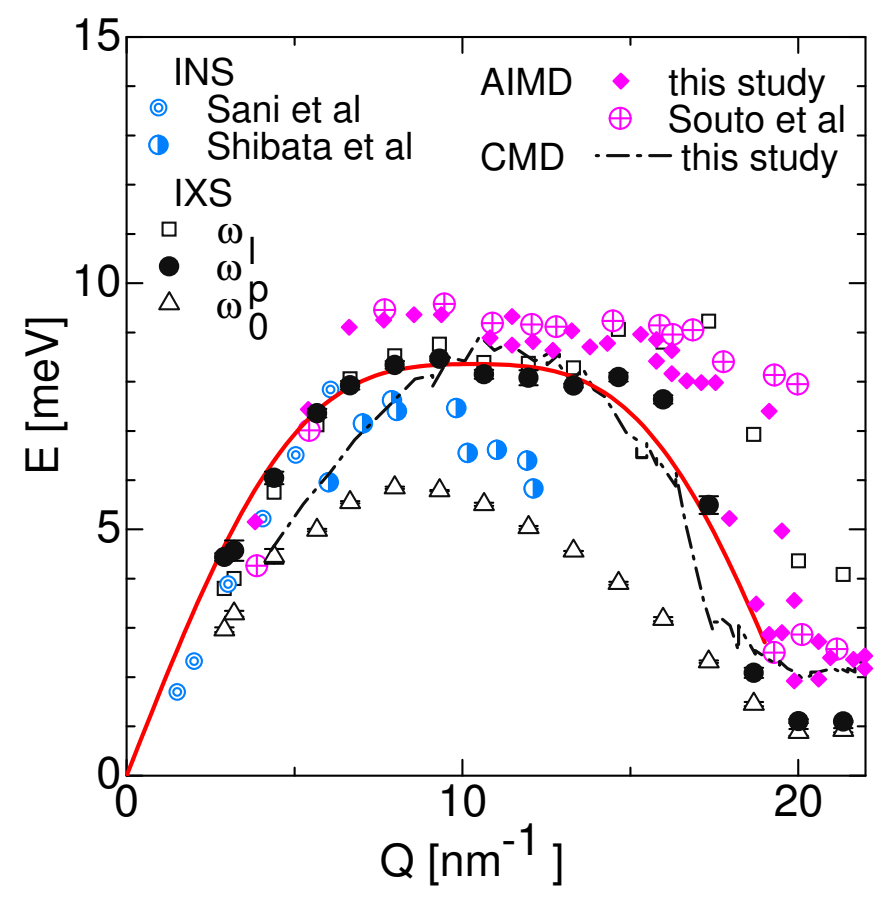

Figure 1. $\omega_{\ell}(Q)$ (open squares), $\omega_{\mathrm{p}}(Q)$ (black circles) and $\omega_{0}(Q)$ (open triangles) obtained by IXS results at $573 \mathrm{~K} . \omega_{\mathrm{p}}(Q)$ by AIMD simulations of this study and that at $600 \mathrm{~K}$ [7] is indicated by diamonds and circles with cross, respectively. Also shown are the excitation energy obtained by INS by Sani $e t$ al. (double circles) and by Shibata et al. (circles with half moon), and CMD (dash-dotted line). A bold red curve denotes the dispersion relation obtained by a regular chain model with two force constants.

wavefunctions was $15 \mathrm{Ry}$. The $\Gamma$ point was only used to sample the Brillouin zone of the MD supercell. We used $128 \mathrm{Bi}$ atoms in a cubic MD cell with periodic boundary conditions. The length of the side of the MD cell was $1.6422 \mathrm{~nm}$. The MD simulation was performed on the ground-state Born-Oppenheimer surface at $573 \mathrm{~K}$ for 32000 steps with a time step of $4.8 \mathrm{fs}$, and reproduced $S(Q, E)$ at $Q \geq 3.8 \mathrm{~nm}^{-1}$ with quality as good as that by Souto et al [7]. We also carried out a CMD simulation at $573 \mathrm{~K}$ using the model potential reported in [5] for a system with 256 particles. To obtain $S(Q, E)$, the simulation was performed for 60000 steps with a time step of 2.4 fs. More details are described in Ref. [13].

\section{Results and discussion}

\subsection{The acoustic dispersion}

The integration of $S(Q, E)$ obtained by IXS was carefully compared with $S(Q)$ obtained independently by our own X-ray scattering experiment. By adjusting the integration at $8 \mathrm{~nm}^{-1}$ with $S(Q)$, we obtained $S(Q, E)$ of the absolute scale. Then $S(Q, E)$ obtained by IXS was compared with that by INS. Our data nicely agree with those by Sani el al. [8]. The difference is that INS spectra are truncated at an energy where inelastic excitations still exist, probably owing to the kinematic constraint of INS. Our results exhibit that the inelastic excitation is 


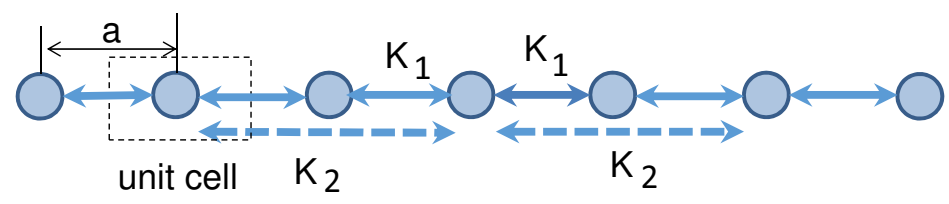

Figure 2. A schematic illustration of a linear chain connected with springs between the first and second nearest neighbors.

visible at $Q \geq 6 \mathrm{~nm}^{-1}$ and it disperses with increasing $Q$. Hence the prediction by Souto $e t$ al. [7] was experimentally confirmed. Details are shown in Ref. [13].

To determine the excitation energy of the acoustic mode, $S(Q, E) / S(Q)$ experimentally obtained should be deconvoluted with the resolution function. We tried three model functions; (a) the memory function formalism with two viscous decay channels [22-25], (b) a model function consisting of single Lorentzian and single damped harmonic oscillator (DHO) [26], and (c) a model function consisting of single Lorentzian and two DHO's. Hereafter, we present the results using (a). Details of these model functions are described in Ref. [13]. Using the memory function formalism, the excitation energy of the acoustic mode, $\omega_{\mathrm{p}}(Q)$, was defined as the peak position of the current-current correlation function deduced from the deconvoluted $S(Q, E) / S(Q)$.

Figure 1 shows $\omega_{\mathrm{p}}(Q)$ of liquid $\mathrm{Bi}$ at $573 \mathrm{~K}$ as a function of $Q$, with the normalized second frequency moment, $\omega_{0}(Q)$, and the normalized fourth frequency moment $\omega_{\ell}(Q)$. $\omega_{0}(Q)$ and $\omega_{\ell}(Q)$ are fitting parameters in the memory function analysis. The figure also depicts the excitation energies obtained by the AIMD and CMD simulations, and those of INS measurements by Sibata et al. [11] and Sani et al. [8]. As shown in the figure, $\omega_{\mathrm{p}}(Q)$ obtained by IXS linearly disperses up to $7 \mathrm{~nm}^{-1}$ This dispersion curve is consistent with that obtained by INS by Sani et al. $\omega_{\mathrm{p}}(Q)$ deviates from the linear dispersion and stays at approximately 8.5 $\mathrm{meV}$ at $10 \leq Q \leq 15 \mathrm{~nm}^{-1}$. A flat region in the dispersion curve is consistent with the prediction by the AIMD simulations by Souto et al. [7] although the AIMD results lie at energies slightly higher than black circles. The IXS results surely confirm the theoretical prediction of a flat-topped dispersion curve.

As shown in Fig.1, the peak positions of the current-current correlation function obtained by CMD (a dash-dotted curve) agree with $\omega_{\mathrm{p}}(Q)$ (black circles) at approximately $10 \mathrm{~nm}^{-1}$. With decreasing $Q$, however, real excitation energies of the acoustic modes deviate much from the CMD results at approximately $5 \mathrm{~nm}^{-1}$. This result suggests that the effective pair potential, able to reproduce characteristics in $S(Q)$, cannot exactly simulate the atomic dynamics in liquid $\mathrm{Bi}$ at low $\mathrm{Q}$.

We could related the flat-topped dispersion curve of the acoustic mode in liquid $\mathrm{Bi}$, using a linear chain model. We describe it using the illustration in Fig. 2. A simple linear chain connected by a spring with force constant $K_{1}$ gives a sinusoidal dispersion curve expressed by $M \omega^{2}=2 K_{1}(1-\cos Q a)$, where $M, \omega$ and $a$ are atomic mass, an excitation energy and a lattice constant, respectively. When the second springs of a force constant $K_{2}$ are fixed between the central atom and the second nearest neighbors as shown in the figure, the equation of the dispersion curve is modified as $M \omega^{2}=2 K_{1}(1-\cos Q a)+2 K_{2}(1-\cos 2 Q a)$. This equation can reproduce a flat-topped dispersion curve by adjusting parameters $K_{1}, K_{2}$ and $a$ [13]. Although the model is based on a regular structure, the concept of a pseudo-Brillouin zone [27] may support applicability of our model to a disordered structure. A linear chain connected by 


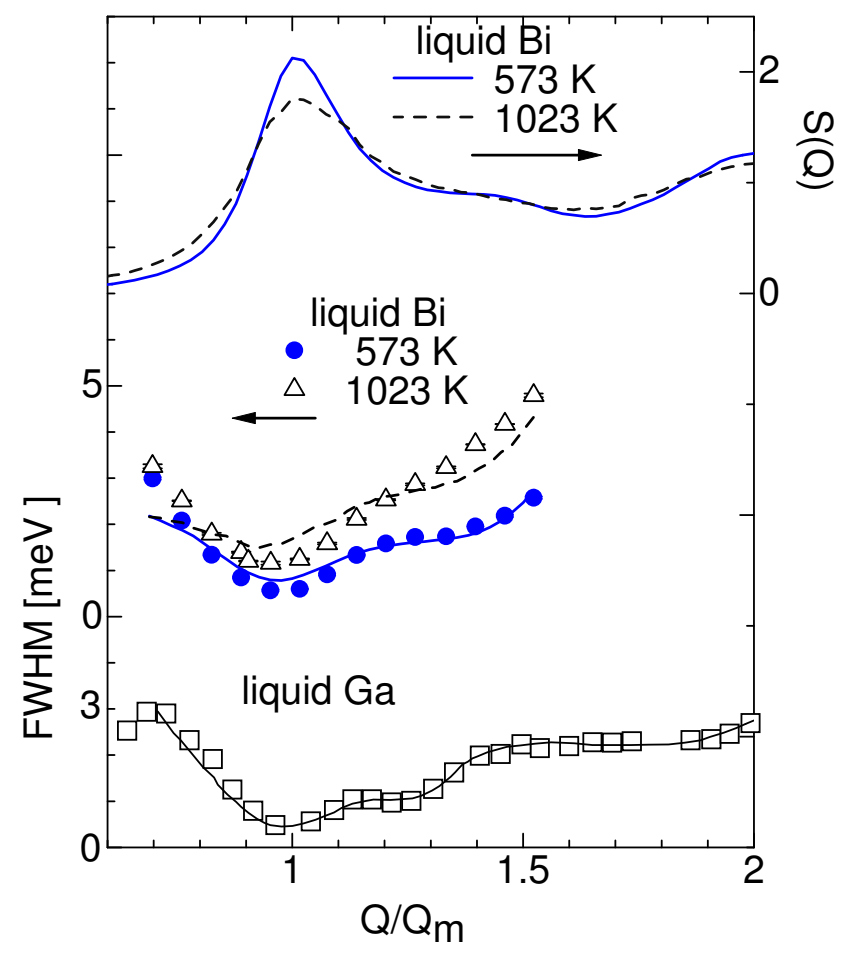

Figure 3. $S(Q)$ (upper curves) and the FWHM of the dynamics structure factor (middle curves and symbols) of liquid Bi at 573 and $1023 \mathrm{~K}$ in this work, and the FWHM of liquid Ga (a bottom curve and symbols) reported in [28]. The FWHM denoted by symbols is the IXS results and that denoted by lines is the theoretical optimization by Eq. (1).

strong and weak springs hints the one-dimensional Peierls distortion. Hence, we could relate the flat-topped dispersion curve with the Peierls distortion in liquid $\mathrm{Bi}$.

\subsection{Hard-sphere-like dynamics}

$S(Q)$ of liquid Bi exhibits the first maximum with a shoulder at higher $Q . S(Q)$ of liquid $\mathrm{Ga}$ exhibits a similar profile. Scopigno et al. [28] carried out IXS measurements of liquid Ga near the melting point, and analyzed the linewidth of $S(Q, E)$ in high $Q$ region including the $S(Q)$ maximum using an Enskog's theory. The equation given by de Schepper and co-workers [29] expresses the linewidth $z_{\mathrm{h}}(Q)$ as,

$$
z_{\mathrm{h}}(Q)=\frac{1}{S(Q)} \frac{D_{\mathrm{E}} Q^{2}}{1-j_{0}(Q \sigma)+2 j_{2}(Q \sigma)},
$$

where $D_{\mathrm{E}}, j_{0}(x)$ and $j_{2}(x)$ are Enskog's diffusion coefficient, zero-th and second order spherical Bessel functions, respectively. $D_{\mathrm{E}}$ is related to Boltzmann diffusion coefficient $D_{0}$ as $D_{\mathrm{E}}=D_{0} / g(\sigma)$, where $g(\sigma)$ is the pair distribution function at a hard sphere diameter $\sigma$. By using free parameters of $D_{\mathrm{E}}$ and $\sigma$, and $S(Q)$ experimentally obtained, Scopigno et al. could reproduce $Q$ dependence of the linewidth well as shown at the bottom of Fig.3. To evaluate 
Table 1. The optimized Enskog's diffusion coefficient $D_{\mathrm{E}}$ and hard sphere diameter $\sigma$ with a packing fraction $\varphi$ and a number density $\rho$.

\begin{tabular}{llllll}
\hline $\mathrm{T}[\mathrm{K}]$ & $D_{\mathrm{E}}\left[\mathrm{m}^{2} / \mathrm{s}\right]$ & $\hbar D_{\mathrm{E}}\left[\mathrm{meV} \mathrm{nm}^{2}\right]$ & $\sigma[\mathrm{nm}]$ & $\varphi$ & $\rho\left[\mathrm{nm}^{-3}\right]$ \\
\hline 573 & $3.6 \times 10^{-9}$ & $2.4 \times 10^{-3}$ & 0.36 & 0.71 & 28.9 \\
1023 & $6.1 \times 10^{-9}$ & $4.0 \times 10^{-3}$ & 0.35 & 0.63 & 27.2 \\
\hline
\end{tabular}

the effective mass, $D_{\mathrm{E}}$ expressed by the packing fraction $\varphi[28,30]$

$$
D_{\mathrm{E}}=\frac{1}{16} \sqrt{\frac{\pi k_{\mathrm{B}} T}{m}}\left(\frac{6}{\pi \rho \varphi^{2}}\right)^{\frac{1}{3}} \frac{(1-\varphi)^{3}}{(1-\varphi / 2)}
$$

was used. Here $m$ and $\rho$ are the effective mass and number density, respectively. In a hard sphere model, $\varphi, \rho$ and $\sigma$ are related as $\varphi=\pi \rho \sigma^{3} / 6$. In the case of liquid $\mathrm{Ga}, \sigma$ slightly larger than the atomic diameter of $\mathrm{Ga}$, and an effective mass sightly larger than the atomic mass were obtained. These results obtained for liquid $\mathrm{Ga}$ were discussed as an indication of existence of dimeric clusters in Ref. [28].

To apply the Enskog's theory, we deconvoluted $S(Q, E) / S(Q)$ with a Lorentzian and the resolution function. Figure 3 shows a full width at half maximum (FWHM) obtained for liquid $\mathrm{Bi}$ at 573 (blue circles) and 1023 (open triangles) $\mathrm{K}$ as a function of $Q / Q_{\mathrm{m}}$, where $Q_{\mathrm{m}}$ denotes the first peak position of $S(Q)$. $Q_{\mathrm{m}}$ values of liquid $\mathrm{Bi}$ and liquid $\mathrm{Ga}$ are 0.21 and $0.25 \mathrm{~nm}^{-1}$, respectively. In our analysis, $S(Q)$ of liquid $\mathrm{Bi}$ was our own data obtained by x-ray diffraction measurements shown at the top of the figure. Bold and broken curves at the middle position of the figure denote the optimized fits using eq.(1), where $D_{\mathrm{E}}$ and $\sigma$ are free parameters as the previous study [28]. As shown in the figure, the best fits reproduce FWHM at $573 \mathrm{~K}$ fairly well but does not at $1023 \mathrm{~K}$. Although FWHM denoted by symbols seems to follow the profile of $S(Q)$, the fitting profiles are clearly worse than the results of liquid $\mathrm{Ga}$. The optimized $D_{\mathrm{E}}$ and $\sigma$ are tabulated in Table 1. $\sigma$ of liquid $\mathrm{Bi}$ is larger than a cutoff distance in $g(r)$, which is approximately $0.29 \mathrm{~nm}$. Asymmetrical bonding consisting of longer and shorter bonds may be inferred in liquid Bi from the optimized $\sigma$. However it was not a reasonable result that the effective mass obtained using eq. (2) was much smaller than the atomic mass.

\section{Conclusion and future perspective}

The present results suggest that a flat-topped dispersion curve observed in liquid $\mathrm{Bi}$ can be explained by taking the interatomic force between the second nearest neighbors into account. This result strongly hints asymmetrical bonding in liquid Bi. Symmetry breaking of bonding related to electronic energy levels in a molecule is well known as the Jahn-Teller effect. Hence, local structure may also be distorted in a liquid if a distortion makes the electronic energy lowered, as the case of liquid Bi.

Peierls distortion in liquid $\mathrm{Bi}$ has been expected by detailed analysis of the average structure [1]. Such a picture was strongly supported by atomic configurations obtained by AIMD simulations AIMD [7], and primitive analysis for the flat-topped dispersion curve confirmed by the present IXS results. It is very important to study the profiles of acoustic dispersion curves in liquid metals and semiconductors where asymmetrical bonding is expected, in order to understand the present results deeply. 


\section{Acknowledgements}

The authors would like to thank the Ministry of Education, Culture, Sports, Science and Technology, Japan, for a Grant-in-Aid for Scientific Research. The synchrotron radiation experiments were performed at the SPring- 8 with the approval of the Japan Synchrotron Radiation Research Institute (JASRI) (Proposals No. 2011B1314, No. 2010B1173, and No. 2009B1283). M. I. would like to thank The Japan Science Society for Sasakawa Grants for Science Fellows (SGSF).

\section{References}

[1] M. Mayo et al., J.Phys:Condens. Matter 25, 505102 (2013).

[2] M. North et al., J. Phys. C 1, 1075-1087 (1968).

[3] Y. Waseda and K. Suzuki, Phys. Stat. Sol. B 49, 339-347 (1972).

[4] Y. Greenberg et al., Euro. Phys. Lett. 86, 36004 (2009).

[5] M. Dzugutov M and U. Dahlborg, Phys. Rev. A 40, 4103-4106 (1989).

[6] W. Jank W and J. Hafner, Phys. Rev. B 45, 2739-2749 (1992).

[7] J. Souto et al., Phys. Rev. B 81, 134201 (2010).

[8] L. Sani et al., J. Non-Cryst. Solids 353, 3139-3144 (2007).

[9] U. Dahlborg and L. G. Olsson, Phys. Rev. A 25, 2712-2720 (1982).

[10] U. Dahlborg and L. G. Olsson, J. Phys. F 13, 555-561 (1983).

[11] K. Shibata et al., J. Phys. Soc. Jpn. 53, 899-902 (1984).

[12] T. Scopigno et al., Rev. Mod. Phys. 77, 881-933 (2005).

[13] M. Inui et al., Phys. Rev. B 92, 054206 (2015).

[14] A. Q. R. Baron et al., J. Phys. Chem. Solids 61, 461-465 (2000).

[15] S. Kohara et al., J. Phys.: Condens. Matter 19, 506101 (2007).

[16] K. Tamura et al., Rev. Sci. Instrum. 70, 144-152 (1999).

[17] S. Hosokawa and W.-C. Pilgrim, Rev. Sci. Instrum. 72, 1721-1728(2001).

[18] $S(Q, E)$ at $1023 \mathrm{~K}$ was consistent with that obtained by AIMD simulations using 512 atoms by M. Ropo et al. [J. Chem. Phys. 145184502, (2016).]

[19] P. Giannozzi et al., J. Phys.:Condens. Matter 21, 395502 (2009).

[20] J. P. Perdew et al., Phys. Rev. Lett. 77, 3865-3868 (1996).

[21] http://www.quantum-espresso.org.

[22] D. Levesque et al., Phys. Rev. A 7, 1690-1700 (1973)

[23] U. Balucani and M. Zoppi Dynamics of liquid State (Clarendon press. Oxford) (1994)

[24] T. Scopigno et al., J. Phys.: Condens. Matter 12, 8009-8034(2000).

[25] F. Bencivenga et al., Phys. Rev. E 75, 051202 (2007).

[26] B. Fåk B and B. Dorner, Physica B 234-236, 1107 (1997).

[27] T. Scopigno et al., Phys. Rev. B 64, 012301 (2001).

[28] T. Scopigno et al., Phys. Rev. Lett. 94, 155301 (2005).

[29] E. G. D. Cohen et al., Phys.Rev. Lett. 59, 2872 (1987).

[30] T. E. Faber Introduction to the theory of liquid metals (Cambridge university press.) 162-165 (1972). 